\title{
Gas Thruster Calibration for Satellite Simulators on an Air Bearing Table
}

\author{
Joshua Cookson \\ Department of Earth and Space Sciences and Engineering \\ York University, M.Sc. Candidate \\ Toronto, Canada
}

\author{
Zheng H. Zhu \\ Department of Mechanical Engineering \\ York University \\ Toronto, Canada
}

\begin{abstract}
This paper covers a simple method of calibration for gas thrusters for the application of simulated space flight. Gas thrusters are a common means of controlling spacecraft, and proper calibration of such thrusters is essential to ensure accurate and stable control of the spacecraft. Satellite simulators attempt to replicate the behaviours of satellites in flight using a 3 degree of freedom air bearing table, which allows for frictionless flight, and gas thrusters to emulate the flight dynamics.
\end{abstract}

Keywords-gas; thruster; calibration; force; spacecraft; satellite; simulator

\section{INTRODUCTION}

In the following we will cover the process of calibrating gas thrusters which are used to control satellite simulators on an air bearing table. The purpose of this is not to precisely determine the output force of the thrusters, but rather to ensure that each thruster outputs the same force. These satellite simulators utilize gas thrusters as their primary means of positional and attitude control. As the thrusters are only able to be toggled on or off during operations, with no adjustments to the amount of output force, the specific output of each thruster must be calibrated to be equal to allow for reliable control under continuous thrust output.

The simulator is a cube with 8 thrusters, each aligned to the horizontal plane, with two thrusters on each vertical face pointing outwards. In this configuration, motion in a straight line relative to a face is controlled by the two thrusters which are positioned in the opposite direction, and rotational maneuvers possible by firing alternating thrusters on each face. The thrusters utilize simple compressed air as propellant. The onboard air tank is charged to 20 Megapascals, this pressure is then reduced through two regulator valves to 0.4 Megapascals which is sent to the thrusters. If the two thrusters do not output the same force, then there will be a torque exerted on the simulator which causes it to turn.

\section{INSTRUMENTATION}

To calibrate the thrusters, a means of detecting the output force is required. For this we utilized a digital force sensor with a magnetic mounting plate to attach the thruster and connected it via USB to a PC to record the measurements. The force sensor used here can resolve forces to an accuracy of 0.001 Newtons.
The thrusters themselves consist of a housing, which contains the actuator to toggle the output, and has a set of power cables running into the base, a set screw which allows the output to be adjusted, and an output nozzle. The set screw has an air inlet coupled with its connection to the housing.

The sensor is placed on a level surface, here using the air bearing platform which is precisely leveled. The sensor is oriented vertically, with the magnetic mount at the top, the thruster is then attached with the nozzle output oriented to either push or pull against the force sensor. Either orientation of the output will provide the same magnitude measurements, with reversed sign of the value. As the thrusters require the power and air lines to be connected to operate, additional lengths of both are added to remove tensional forces. The air line is then charged to the operating pressure as the line may move while it pressurizes. This configuration on the level surface allows us to easily consider the force due to gravity acting on the thruster and the connected lines and assume the only change in force measured is due to the thruster output. The sensor is then zeroed in this position.

\section{MEASUREMENTS}

The sample rate for the sensor was set to 200 samples per second for a 10 second duration. Force measurements were taken for a 1-3 second duration with the thruster off, and it is then switched on for the remainder of the test. Fig. 1 shows a single set of data. There is a large spike in the measured force

when the thruster is switched from off to on, this is due to the actuation of the control valve in the thruster. This region of data is not included in the force determinations as it does not

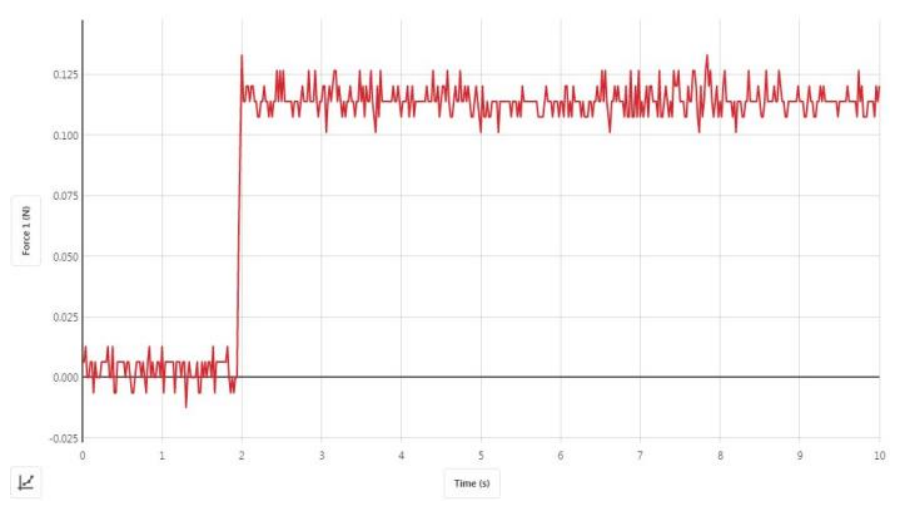

Figure 1. Force Output Data 
represent the steady state output of the thruster.

The data is then averaged for both the off and on positions of the thruster, the averaging process reduces the contributions of noise and vibrations in the system due to the air flow generated by the thruster, thereby providing a more accurate representation of the steady state characteristics of the system. The average value for the off state of the thruster, representing the zeroing error and noise of the system, is then subtracted from the average value of the on state of the thruster. This final value represents the steady state output force of the thruster.

To calibrate each thruster to the same force, the performance envelope for each thruster nozzle first needs to be determined. Each nozzle is hand made and therefore has unique performance characteristics. The output force is measured with the setscrew at maximum and minimum in turn, which gives the range of pressures which are attainable with that thruster.

Following the determination of the performance envelopes and selection of thrusters to calibrate, an overlapping force is selected for each to be calibrated to, here being 0.065 Newtons. To reach this output each thruster is again mounted on the force sensor, the setscrew is adjusted slightly, and another set of measurements is taken. This process is repeated until the desired output force is reached, the setscrew is then locked in place with a nut. Table 1 shows the resulting calibration measurements.

TABLE I. ForCE CALIBRATION RESUlts

\begin{tabular}{|l|l|l|l|}
\hline \multirow{2}{*}{ Thruster } & \multicolumn{3}{|c|}{ Force Measurement } \\
\cline { 2 - 4 } & Measured $(\boldsymbol{N})$ & Zero Error $(\boldsymbol{N})$ & Force Output $(\boldsymbol{N})$ \\
\hline 1 & 0.065 & 0 & 0.065 \\
\hline 2 & 0.074 & 0.009 & 0.065 \\
\hline 3 & 0.060 & -0.005 & 0.065 \\
\hline 4 & 0.068 & 0.003 & 0.065 \\
\hline 5 & 0.066 & 0.001 & 0.065 \\
\hline 6 & 0.073 & 0.008 & 0.065 \\
\hline 7 & 0.065 & 0 & 0.065 \\
\hline 8 & 0.067 & 0.002 & 0.065 \\
\hline
\end{tabular}

\section{VERIFICATION}

The final calibration of the thrusters is then able to be verified through a flight test of the satellite simulator system. The simulator is tasked to travel in a straight line and then stop. This requires 2 thrusters to be fired to begin moving, and then fire the 2 alternate thrusters on the same faces to bring the simulator to a stop. With the thrusters properly calibrated the simulator can perform this maneuver with no rotation occurring.

\section{CONCLUSION}

Gas thrusters are a common means of spacecraft control. Using this method of thruster calibration, the satellite simulator system can reliably demonstrate the dynamics of space flight, with stable control using gas thrusters. This method of thruster calibration proves to be simple and reliable for this application.

\section{REFERENCES}

[1] J. Jarrige, P. Thobois, et. All, "Thrust measurements of the Gaia mission flight-model cold gas thrusters," Journal of Propulsion and Power, vol. 30, issue 4, pp 934-943, August 2014.

[2] SF. Wu, WH. Steyn, RE. Bordany, "In-orbit thruster calibration techniques and experiment results with UoSAT-12," Control Engineering Practice, vol. 12, issue 1, pp. 87-98, January 2004.

[3] J. Lun, C. Law, "Direct thrust measurement stand with improved operation and force calibration technique for performance testing of pulsed micro-thrusters," Measurement Science and Technology, vol. 25, issue 9, article 095009, September 2014. 